\title{
Multicavity linear transformer driver facility for $Z$-pinch and high-power microwave research
}

\author{
B. J. Sporer $\odot,{ }^{1}$ A. P. Shah $\odot,{ }^{1}$ G. V. Dowhan $\odot,{ }^{1}$ R. V. Shapovalov $\odot,{ }^{1}$ D. A. Packard, ${ }^{1}$ \\ M. Wisher, ${ }^{2,}$ J. J. Leckbee, ${ }^{2}$ K. J. Hendricks $\odot,{ }^{3}$ B. W. Hoff $\odot,{ }^{3}$ Y. Y. Lau, ${ }^{1}$ \\ R. M. Gilgenbach, ${ }^{1}$ N. M. Jordan, ${ }^{1}$ and R. D. McBride ${ }^{1}$ \\ ${ }^{1}$ Nuclear Engineering and Radiological Sciences, University of Michigan, \\ Ann Arbor, Michigan 48109, USA \\ ${ }^{2}$ Sandia National Laboratories, Albuquerque, New Mexico 87185, USA \\ ${ }^{3}$ Air Force Research Laboratory, Albuquerque, New Mexico 87117-5776, USA
}

(Received 27 July 2021; accepted 21 September 2021; published 21 October 2021)

\begin{abstract}
BLUE is a four-cavity linear transformer driver system in the Plasma, Pulsed Power, and Microwave Laboratory at the University of Michigan. Each BLUE cavity can store up to $2 \mathrm{~kJ}$ of electrical energy and provide an open-circuit voltage of $200 \mathrm{kV}$ when using the maximum bipolar charge voltage of $\pm 100 \mathrm{kV}$. The impedance of each cavity is approximately $0.5 \Omega$, and the rise time of the current pulse into a reasonably matched load is $\sim 100 \mathrm{~ns}$. The system will be used to study high-energy-density $Z$-pinch plasmas and high-power radiation sources. The facility has been equipped to handle intense bursts of $\mathrm{x}$ rays, nuclear fusion neutrons, and high-power microwaves (HPM). This paper reports on the construction and performance of the first (prototype) BLUE cavity and its auxiliary systems, as well as preliminary experiments using the cavity to drive resistive loads and a magnetically insulated line oscillator (MILO) HPM device. In these initial single cavity, BLUE-driven MILO experiments, $1.19 \mathrm{GHz}$ microwave oscillations were successfully generated.
\end{abstract}

DOI: 10.1103/PhysRevAccelBeams.24.100402

\section{INTRODUCTION}

Linear transformer drivers (LTDs) [1-4] are an innovative and compact pulsed power technology under extensive investigation around the world [5-22]. At the University of Michigan, a single-cavity LTD facility called MAIZE has been operational for over a decade $[7,18,19]$. MAIZE is a very low-impedance driver $(\sim 0.1 \Omega)$ and is capable of generating a current pulse that rises from 0 to $1 \mathrm{MA}$ in as little as $100 \mathrm{~ns}$ into a matched load. To complement the single-cavity MAIZE facility, a four-cavity LTD facility called BLUE is under construction. The 1.25-m diameter cavities used for BLUE were originally fabricated at the Institute of High Current Electronics (IHCE) in Tomsk, Russia, before becoming the basis for the 21-cavity Ursa Minor facility $[12,13]$ at Sandia National Laboratories (SNL). Ursa Minor has since been dismantled and

\footnotetext{
Present address: SARA, Inc., Colorado Springs, Colorado 80920, USA.

Published by the American Physical Society under the terms of the Creative Commons Attribution 4.0 International license. Further distribution of this work must maintain attribution to the author(s) and the published article's title, journal citation, and DOI.
}

repurposed. The original four IHCE cavities are now part of the BLUE facility at the University of Michigan.

Figure 1 shows the BLUE experimental bay in the Plasma, Pulsed Power, and Microwave Lab (PPML) at the University of Michigan. A concrete brick wall was arranged around the cavities to contain radiation produced by the facility, including neutrons from deuterium pinches. The white steel rack suspends the cavities on their sides and allows the cavities to be stacked in series. The rack has rails that allow the cavities to glide into position. A motorized overhead crane can remove individual cavities and place them on a purpose-built wooden stand for servicing. Either 1, 2,3 , or 4 cavities can be stacked in series so that the driver impedance and driver voltage can be varied, and the effects of this variation on load performance can be investigated. The coupling of many stacked LTD cavities is important for future conceptual accelerators like Z-300 and Z-800 [15], which will require thousands of LTD cavities combined in both series and parallel configurations.

The donation from SNL included the empty metal cavities themselves as well as some of the fundamental internal elements, such as the spark-gap switches, the capacitors, the insulators, the anode transmission line framework, and the Metglas cores. Auxiliary equipment had to be built from scratch, including the high-voltage charging circuitry, the control systems, the trigger pulse generator, and the core reset or pre-magnetization pulse generator. 


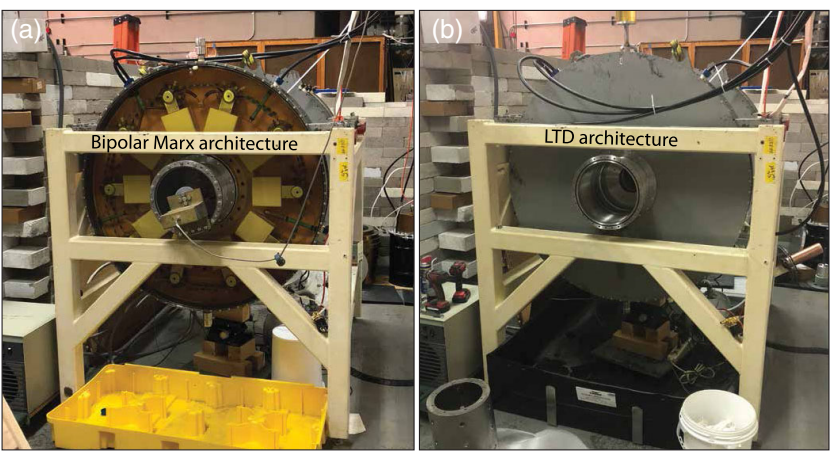

FIG. 1. The BLUE experimental bay in the Plasma, Pulsed Power, and Microwave Lab (PPML) at the University of Michigan, with the prototype BLUE cavity configured as: (a) a bipolar Marx generator with a clear plastic lid; and (b) as a linear transformer driver with a steel lid.

The remainder of this paper is organized as follows. In Sec. II, the assembly of the first (prototype) BLUE LTD cavity is described. In Sec. III, the auxiliary systems are described. In Sec. IV, results are presented from experiments where the cavity was fired in two architectures: (1) as a single-stage bipolar Marx generator without ferromagnetic cores [Fig. 1(a)] and (2) as a true LTD with ferromagnetic cores and a pre-magnetization pulse generator to reset or magnetize those cores [Fig. 1(b)]. Both architectures were tested with a dummy resistive load. In Sec. V, results are presented from experiments where BLUE was used to drive a high-power microwave (HPM) device known as a magnetically insulated line oscillator (MILO). In these BLUE-driven MILO experiments, 1.19-GHz microwave oscillations were successfully generated.

\section{THE PROTOTYPE BLUE CAVITY}

Images of the first completed BLUE cavity are presented in Figs. 1 and 2. In Figs. 1(a) and 2, a clear polycarbonate lid was in use, which enables the interior components to be observed during operation. The plastic lid also eliminates the parasitic current path around the LTD case [19], and thus all of the current is driven through the load at the center of the machine. Because the parasitic current path is eliminated, ferromagnetic cores were not required nor installed in Figs. 1(a) and 2 (i.e., the cavity was in a bipolar Marx generator configuration). As our testing evolved, the clear plastic lid was eventually replaced with a metal lid, and thus pre-magnetized ferromagnetic cores became a requirement (see Sec. III E for a discussion on the LTD cores).

Referring to Fig. 2, each BLUE cavity is comprised of ten "bricks," where each brick consists of two $100-\mathrm{kV}$, 20-nF capacitors (general atomics part \#35467) and a single 200-kV ( $\pm 100-\mathrm{kV})$ spark-gap switch (L3Harris Model \#40264-200kV). A photograph of an L3Harris spark-gap switch is presented in Fig. 3. Each switch was refurbished

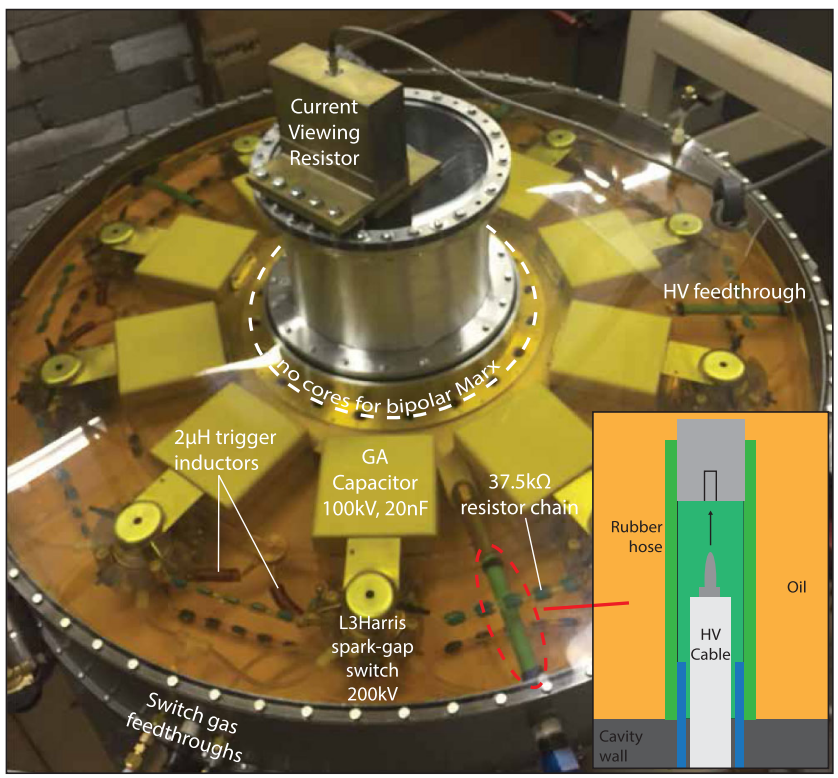

FIG. 2. The first assembled BLUE cavity, shown here with a clear polycarbonate lid and no ferromagnetic cores (i.e., the cavity is in a single-stage bipolar Marx generator configuration). The cavity is filled with transformer oil. The small vacuum chamber on top of the cavity houses the resistive load. The brass structure on top of the chamber is the current viewing resistor (CVR). Plug-in feedthroughs with $3 \mathrm{D}$-printed parts allow high-voltage cables to be easily disconnected from the cavity without removing the lid or draining the transformer oil.

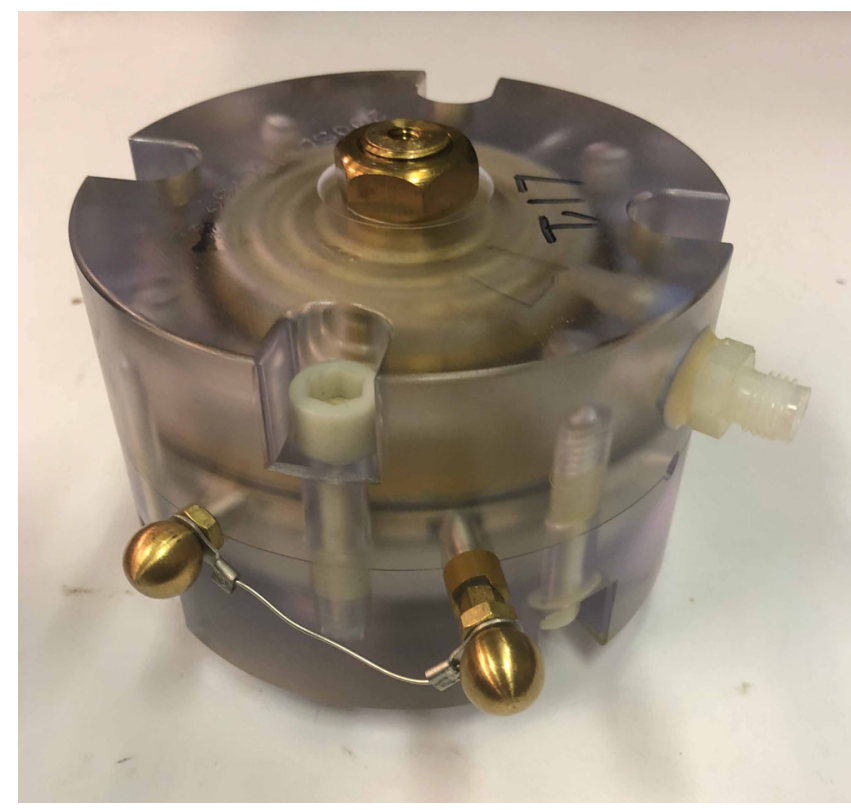

FIG. 3. An L3Harris spark-gap switch with brass electrodes, capable of operation at $200 \mathrm{kV}( \pm 100 \mathrm{kV})$ and up to 200 psi gas fill. These switches are used in the MAIZE \& BLUE LTD systems at the University of Michigan. In this image, the switch is shown with a shorting wire between the UV-pin and midplane. In the BLUE cavity, the shorting wires are replaced with 3-M $\Omega$ resistor chains for proper operation of the UV-pin. 
and tested with ten nominal discharges in the PPML "bricktester" before installation in the BLUE cavity. Switch prefires were rarely observed in either the brick tester or in BLUE - only one definite pre-fire event was confirmed in the $\sim 100$ shots performed thus far on the first cavity.

Each BLUE cavity also consists of a transmission line framework for mounting the bricks in the cavities, large plastic insulator disks for separating the anode and cathode sides of the cavity, charging and triggering circuitry (such as HV resistors, inductors, and cable feedthroughs), switch gas lines, and diagnostics.

The trigger inductors (each $\sim 2 \mu \mathrm{H}$ ) were installed between the main trigger bus line and the UV-pins/midplanes of the L3Harris switches. Due to the finite capacitance of a switch midplane $(\sim 7 \mathrm{pF})$, the trigger inductors serve to amplify the trigger pulse and reduce the jitter of the switch [20]. They also provide some isolation between switches during the breakdown process. The bronze-colored trigger inductors can be seen attached to the switches in Fig. 2.

Bipolar LTD cavities such as MAIZE and BLUE require both a positive and negative high-voltage charging supply. The open-circuit output voltage of the cavity is equal to the difference between the positive and negative values. Since these values usually have the same magnitude, the opencircuit output voltage is usually twice the "charge voltage." For example, a $\pm 100-\mathrm{kV}$ charge voltage results in a $200-\mathrm{kV}$ open-circuit output voltage. The spark-gap switches are bipolar as well, holding off both charge polarities from each other, until a trigger pulse on the grounded midplane electrode breaks down the switch. Each L3Harris switch also includes a "UV-pin." Upon triggering, the pin creates a small spark at the switch midplane. The spark floods the switch gas with photoionizing UV light, thus reducing the breakdown jitter [23,24]. In a typical bipolar LTD cavity, the switch electrodes (and charging side of the capacitors) are chained together around the cavity through charging resistors or charging inductors (see Fig. 2).

A high "rep-rate" (rate of repetitively charging and firing) is desired for various experimental platforms on BLUE (e.g., HPM experiments and dense plasma focus neutron source experiments). Thus, dual Spellman 12-kW power supplies were acquired. They allow a theoretical reprate $>1 \mathrm{~Hz}$, as long as the charging impedance is kept low. However, having a small charging impedance between each brick may lead to degradation of the L3Harris spark-gaps during pre-fire events, due to excessive current draw from neighboring bricks. Resistor chains were assembled to serve as the charging impedance between each brick. Ultimately, a $37.5-\mathrm{k} \Omega$ total resistance for each chain was chosen as a compromise between brick isolation and fast charging time. These resistor chains limit undesired pre-fire currents through adjacent bricks to $<2.7 \mathrm{~A}$. Considering the 20-nF capacitors used in BLUE, the $R C$ time constant of this charging impedance is $750 \mu \mathrm{s}$.
Five high-voltage cables are fed into each BLUE cavity: one for each charge polarity, one for a trigger pulse, and one for each pre-magnetization pulse polarity. Since the BLUE cavities must be moved back and forth between the servicing test stand and the side-mounting stacking rack, it was desired for the high-voltage (HV) cables to be able to be unplugged from the cavity without the need to open the cavity or drain the transformer oil. To this end, a novel plug-in connection was developed utilizing SLA 3D-printed parts. This HV feedthrough connection is depicted in Fig. 2. The cavity dimensions limited the length of the feedthroughs, making the dc charge cables especially prone to arcing. Luckily, it was found that the arcing could be eliminated by partially filling the feedthroughs with a dielectric grease.

\section{AUXILIARY SYSTEMS}

\section{A. Control panel}

To streamline the firing sequence of the BLUE system, a consolidated and intuitive control interface was desired. The firing sequence includes monitoring safety interlocks, operating the Spellman high-voltage power supplies, operating high-voltage Ross relays for isolation or grounding, and generating the fire command logic pulse. Semiautonomous control of the firing sequence and other system functions minimizes manual input and allows for faster rep-rating of the facility.

Figure 4 shows the control panel assembled for BLUE. The panel is semiautonomous, using the programmable Arduino Uno board (which is based on the ATmega328P 8-bit microcontroller). The best electrical resilience was obtained with the Ruggeduino-SE, a ruggedized version of the Uno manufactured by Rugged Circuits.

The panel is capable of rep-rating BLUE by automatically controlling the charging sequence as fast as possible. The only user input required is pressing the button to fire the machine. The panel has many other features as well, including monitoring for pre-fire events, automatic purging

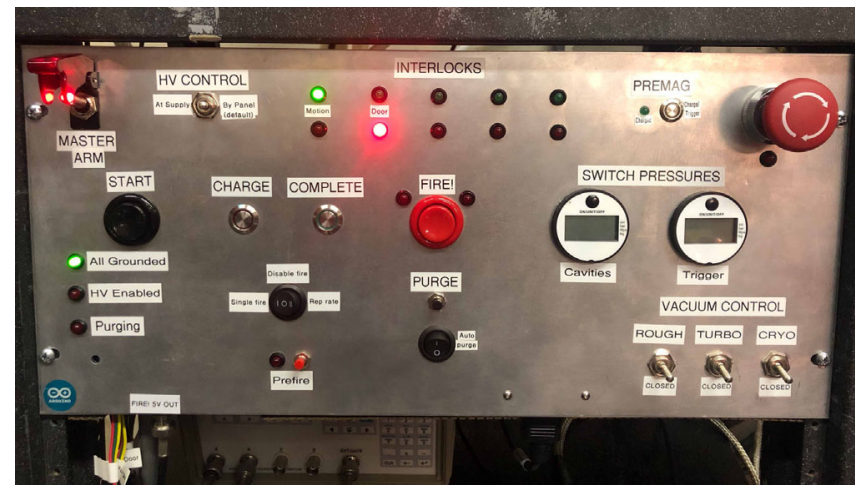

FIG. 4. Arduino Uno (ATmega328P-based) control panel for semiautonomous control of the BLUE charging and firing sequence, as well as other system functions. 


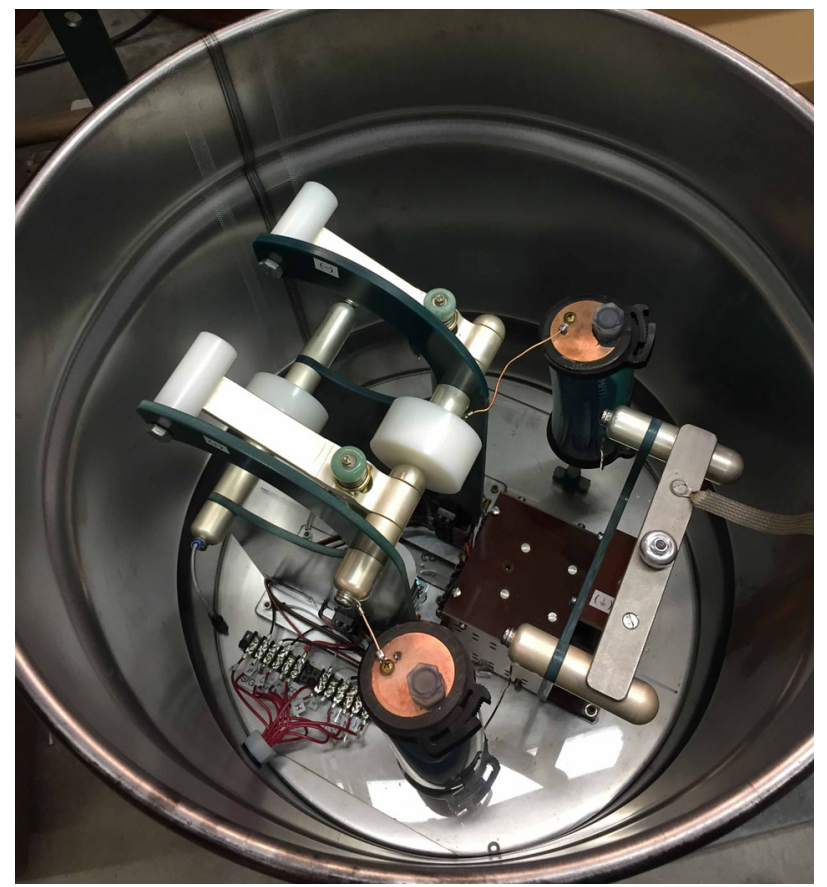

FIG. 5. A 20-gallon oil drum contains the three Ross HV relays used in the charging circuit. The relays are operated by the control panel (see Sec. III A and Fig. 4) during the charging sequence. The large copper-sulfate dump resistors in this photograph were ultimately replaced with $50-\mathrm{k} \Omega$ ceramic resistors.

of the switch gas, and control of the pre-magnetization pulse generator.

\section{B. HV Ross relay barrel}

Three high-voltage relays (produced by Ross Engineering Corporation) are used to connect or disconnect the cavity capacitors from the charging supplies. They also provide passive grounding in the event of an emergency abort or unexpected power loss. Operation of the three relays is handled by the Arduino-based control panel. The relays must be submerged in transformer oil to prevent arcing, so a 20 -gallon steel drum was used to house the three relays, as shown in Fig. 5. The drum also contains two $50-\mathrm{k} \Omega$ ceramic dump resistors, which absorb the energy stored in the cavity(s) upon abort. High-voltage feedthroughs similar to those developed for the prototype BLUE cavity allow up to five positive and five negative cables to plug into the barrel.

\section{200-kV trigger pulse generator}

The L3Harris spark-gap switches require a high-voltage pulse as a trigger. The pulse is applied to the midplane, distorting the field in the switch enough to breakdown the pressurized gas (dry "zero" air) in the switch. A very fast $\frac{d V}{d t}$ on the rising edge of the pulse is desired to minimize the jitter of a single switch and the jitter between multiple switches receiving the same trigger pulse. The pulse should also have a macroscopic amount of energy $(\gg 1 \mathrm{~J})$ to ensure robust triggering of many switches in parallel (up to 40 switches with all four BLUE cavities in operation).

To achieve a large $\frac{d V}{d t}$, a single bipolar brick, charged in parallel with the LTD cavities, is used to trigger the cavities. This gives a trigger voltage equal to twice the charge voltage (also equal to a single-cavity's open-circuit output voltage). The trigger brick is housed in a ten-gallon steel drum with high-voltage plug-in feedthroughs similar to those used on the prototype BLUE cavity and Ross relay barrel. Positive or negative trigger output may be chosen by grounding the opposite polarity. The trigger pulse generator barrel is shown in Fig. 6.

The brick used in the trigger generator is identical to those used in the BLUE cavities, consisting of two 20-nF capacitors and a L3Harris spark-gap switch. The single spark-gap switch in the trigger generator is itself triggered by a PT55 pulse generator, which is mounted to the top of the barrel, as shown in Fig. 6. See Sec. III D for further discussion of the PT55.

\section{300-V pulse generator (PT003 replacement)}

The Pacific Atlantic Electronics (PAE) PT55 module (see Fig. 7), which is no longer in production, uses a rare krytron switch to produce a $+50 \mathrm{kV}$ output pulse with a risetime of less than $2 \mathrm{~ns}$. The module requires a low-power $+7 \mathrm{kVDC}$ charge voltage and a positive 250-300 V trigger pulse. PT55s are compact, low-jitter, and very useful in a

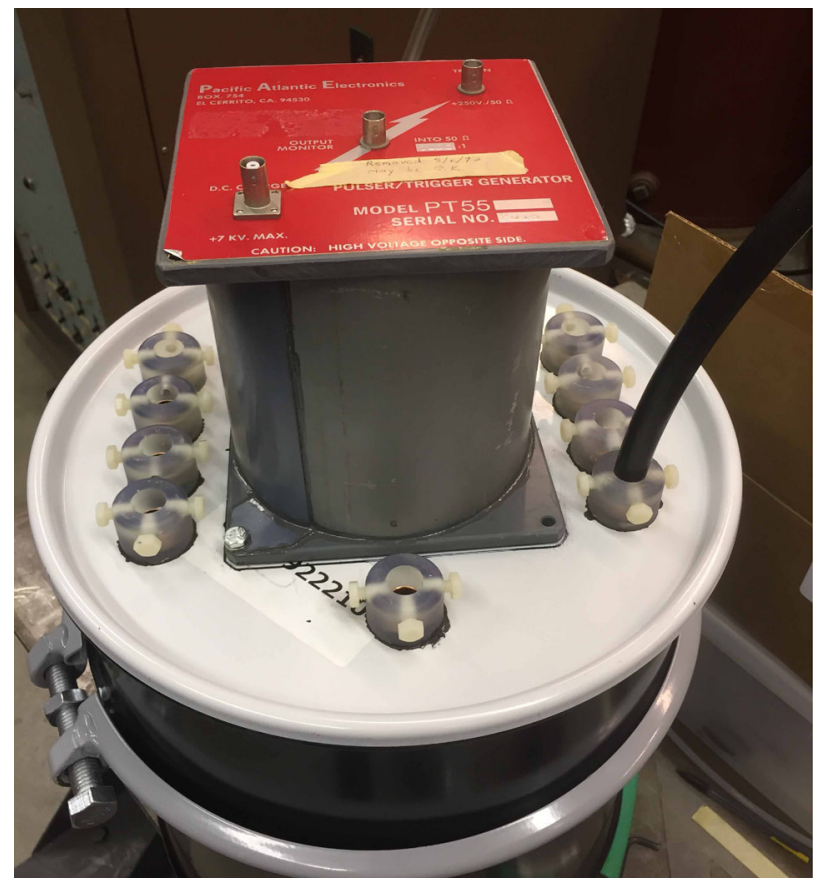

FIG. 6. The 10-gallon oil drum containing the trigger pulse generating brick, which is charged in parallel with the BLUE LTD cavities and triggered by the PT55 pulse generator mounted to the top of the drum. 


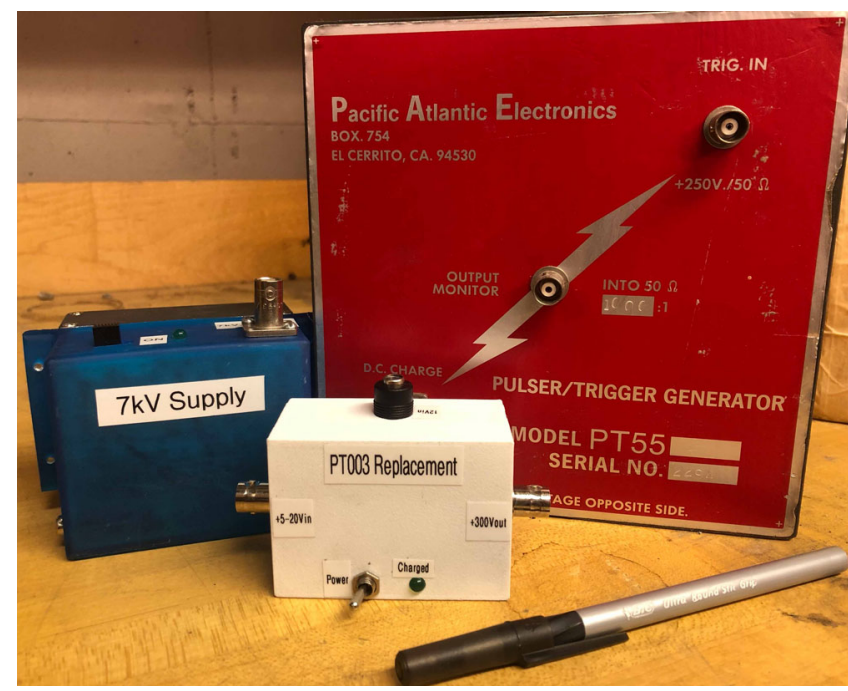

FIG. 7. The Pacific Atlantic Electronics PT55 module with a +7 kVDC power supply and the newly developed $+300 \mathrm{~V}$ pulse generator (PT003 replacement). Together, these modules can produce $\mathrm{a}+50 \mathrm{kV}$ output pulse with $<10$-ns jitter from a logiclevel $(+5 \mathrm{~V})$ input pulse.

pulsed-power laboratory, namely for triggering spark-gap switches. The single L3Harris spark-gap switch used in the BLUE trigger pulse generator (and also used in the MAIZE \& BLUE LTD cavities) can be reliably triggered by a PT55 pulse, even when the switch is being operated at full voltage $(200 \mathrm{kV})$. Several PT55s of varying condition are stockpiled in the PPML.

The PT55 draws $<1 \mathrm{~mA}$ from its $+7 \mathrm{kVDC}$ power supply, so the charge voltage can be provided by a small $12-\mathrm{V}$ dc-dc module (see Fig. 7). By contrast, the PT55's positive $250-300 \mathrm{~V}$ input trigger pulse is not so trivial to produce. Thus, the defunct PAE company also offered the PT003, a small solid-state device that, when given a +100 VDC charge voltage and a logic-level $(+5 \mathrm{~V})$ trigger pulse, produces a positive $250-300 \mathrm{~V}$ output pulse suitable for triggering the PT55. For minimal jitter, the PT003 had a $<10$-ns risetime, as shown in Fig. 8.

Unfortunately, the PPML supply of PT003s has dwindled much faster than the supply of PT55s. Since PT55s continue to be used around the lab, including for the BLUE trigger pulse generator, a suitable replacement for the PT003 had to be procured. With no inexpensive commercial options, the replacement module described in Figs. 7-9 was developed, with inspiration from the $200-\mathrm{V}$ spark-gap trigger circuit in Ref. [25].

The circuit of the new $+300 \mathrm{~V}$ pulse generator is shown in Fig. 9. Six small film capacitors in parallel, totaling $6 \mathrm{nF}$, are charged to $-300 \mathrm{~V}$ with a small, commercially available $12-\mathrm{V}$ dc-dc module. Five enhancement-type, $P$-channel MOSFETs (PMOS) in parallel (Nexperia BSP230,135) are used as a low-side switch to rapidly connect the $-300 \mathrm{~V}$ to ground when triggered. Multiple capacitors and PMOS elements are used in parallel to minimize the

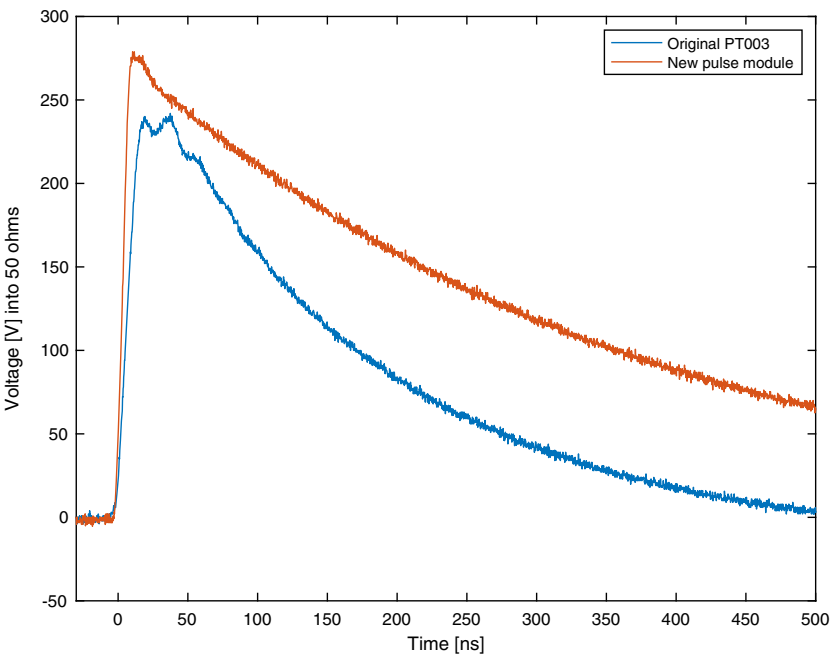

FIG. 8. Output pulses from an original PAE PT003 (blue) and the new $+300 \mathrm{~V}$ pulse generator (orange) into $50 \Omega$, when given $\mathrm{a}+5 \mathrm{~V}$ TTL trigger signal.

inductance and resistance of the circuit. Triggering the PMOS elements produces a $+300 \mathrm{~V}$ pulse into $50 \Omega$ on the opposite side of the capacitors. The over-damped pulse has an approximately 200-ns $e$-folding decay time, similar to the original PT003 (see Fig. 8).

To trigger the PMOS elements in this configuration, a negative voltage pulse must be applied to the gates. It was found in testing that a positive $10-20 \mathrm{~V}$ pulse, produced by a commercial pulse generator, could be inverted with a low leakage-inductance pulse transformer, and thus trigger the PMOS elements. However, the gate input capacitance of the PMOS elements, with several in parallel, is significant. The inverted pulse from the pulse generator, even at the maximum $+20 \mathrm{~V}$ amplitude, was unable to draw enough

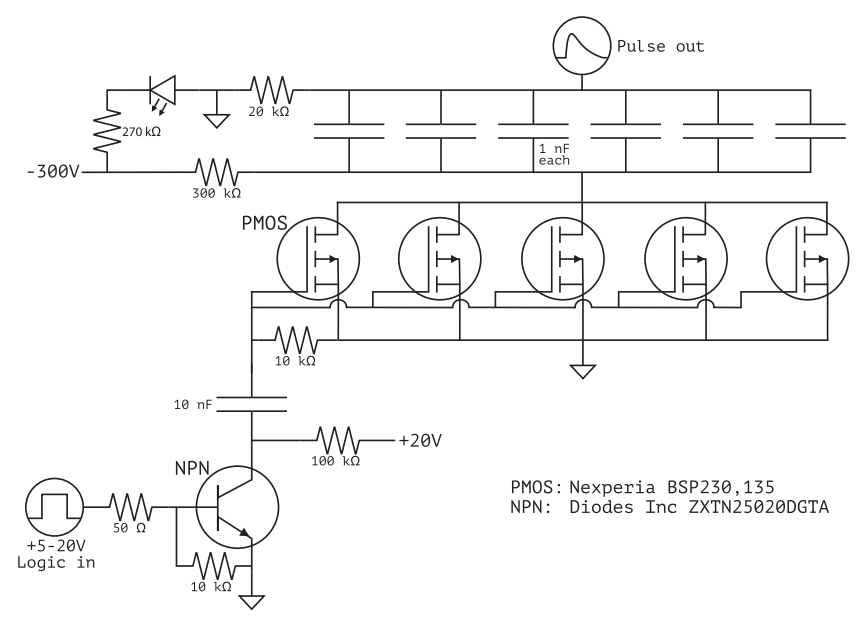

FIG. 9. Circuit diagram of the $+300 \mathrm{~V}$ pulse generator designed to trigger the PT55 mounted to the BLUE trigger pulse generator. The $+20 \mathrm{~V}$ and $-300 \mathrm{~V}$ are provided with small $12 \mathrm{~V}$ dc-dc converters. This generator replaces the defunct PT003 module. 
instantaneous current from the gates to achieve the desired risetime and amplitude.

To trigger the PMOS elements more strongly with only a logic-level $(+5 \mathrm{~V})$ pulse, an NPN transistor (Diodes Inc. ZXTN25020DGTA) was added to the circuit. A 10-nF capacitor is charged to $+20 \mathrm{~V}$ from an additional dc-dc converter. This capacitor is discharged to ground via the transistor upon receipt of a positive logic-level pulse. Closure of the transistor switch produces a $-20 \mathrm{~V}$ pulse on the output of the capacitor, which is fed to the PMOS gates. The addition of the NPN transistor to the circuit produced the desired amplitude and risetime to reliably trigger the PT55 with jitter $<10$ ns (see Fig. 8).

\section{E. Pre-magnetization pulse generator for resetting BLUE's ferromagnetic cores}

In an LTD cavity, there is a parasitic path that the discharge current can take along the inner surface of the LTD's metallic case [19]. This parasitic path is in parallel with the load. To limit the current that takes this parasitic path (and thus force the current through the load as desired), LTD cavities usually include two ferromagnetic cores (an upper core and a lower core) within the loop formed by the parasitic current path.

The ferromagnetic cores typically consist of thin iron or Metglas tape, which is laminated with a dielectric film, wound into large rings, and potted in epoxy resin. Since the parasitic current path encloses the cores, generation of a parasitic current drives magnetization of the cores. Since the cores are made of thin strips of semiresistive material with high-magnetic permeability $\mu \gg \mu_{0}$, there is a high inductance associated with the parasitic path, which minimizes the fraction of current taking this path. In addition, there is a resistive effect due to eddy currents generated during magnetization of the cores, which appears at high frequency [11]. Energy losses in the cores during an LTD discharge are often modeled with a static (and many times empirically determined) resistance in parallel with the load. To model lower-frequency pulses around the cores, such as the core resetting or pre-magnetization discharge, an initial resistance that reduces rapidly after several microseconds is appropriate.

Due to magnetic hysteresis in the cores, the parasitic current from repeated cavity discharges eventually saturates the magnetic domains in the cores in a direction that reduces the effective permeability of the cores and thus reduces the impedance of the parasitic current path. To recover the maximum effective impedance for the parasitic current path, the alignment of the magnetic domains must be reset (reversed) back into their ideal direction for an LTD discharge. This can be done by applying a pre-magnetization pulse to the cavity. This "pre-mag" pulse drives a current in the LTD's metallic case that runs in a direction opposite to that of the parasitic current generated during an LTD discharge. The pre-mag pulse can be applied using a

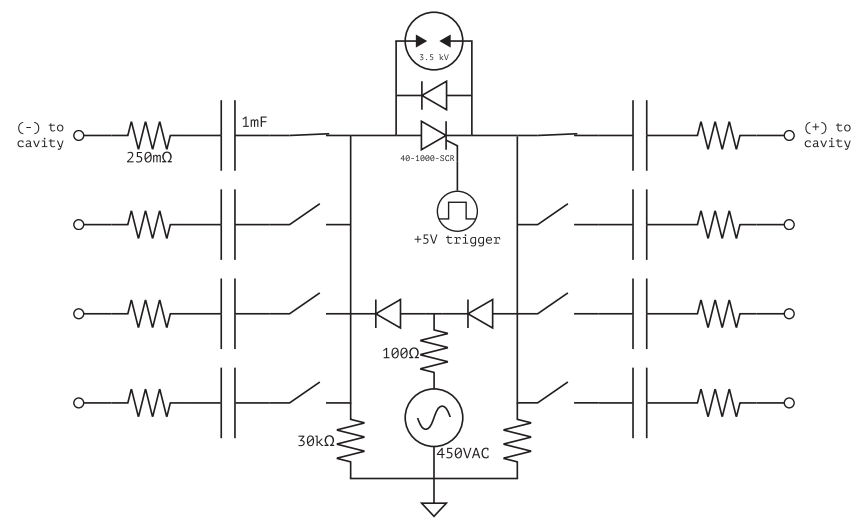

FIG. 10. Circuit diagram of the pre-magnetization pulse generator for resetting the ferromagnetic cores on BLUE. In this diagram, only the output to the first cavity is enabled.

much lower voltage and generating a much lower peak current than that of an LTD discharge, but the pre-mag pulse must then be applied over a proportionally longer timescale. The pre-mag pulse can be applied anytime between LTD discharges, but it is typically done before installation of the experimental load, since the pre-mag pulse can ruin sensitive loads by driving modest currents through the load over long timescales.

The "volt-second product" of the cores is the parameter that defines the magnetic saturation properties of the cores. It can be roughly calculated from the material properties and geometry of the cores. From previous literature on the cavities (during the development of Ursa Minor) [26], the volt-second product of the cores used in the BLUE cavities is estimated to be around $20 \mathrm{mV} \mathrm{s}$. This figure makes intuitive sense: one can think about the volt-second product as if each core must "hold-off" the $100-\mathrm{kV}$ pulse from half of the cavity for at least $200 \mathrm{~ns}$ (the typical experimental time). It also gives an estimate of the required pre-mag pulse duration for saturation. For example, if the pre-mag pulse voltage is $600 \mathrm{~V}$, then saturation can be expected after a pulse duration of $33 \mu \mathrm{s}$. Reference [26] also recommends a pre-magnetizing current of at least $1.5 \mathrm{kA}$ to reach saturation in the Ursa Minor (or BLUE) cores.

After exploratory modeling in LTspice, a relatively simple $R C$ discharge was chosen to generate the required pulse while minimizing cost and complexity of the pre-mag system. As shown in Fig. 10, eight resistor-capacitor combinations are needed to provide a positive and negative channel for each of the four cavities. The capacitors (which can be individually disconnected from the circuit via screwin breakers) are charged by a double-rectified 450-VAC transformer to (theoretically) $\pm 636 \mathrm{~V}$. In reality, the capacitors charge to about $610 \mathrm{~V}$ due to leakage through the passive dump resistors. After charging is complete, a high-power, push-pull thyristor (Behlke HTS 40-1000SCR) discharges the positive and negative channels to each other in a low-side bipolar fashion, similar to the 


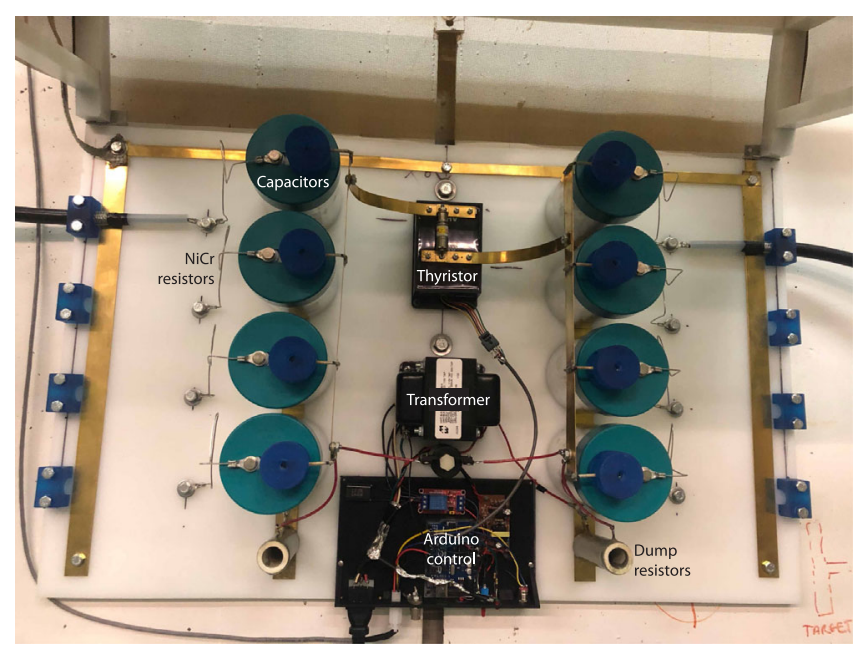

FIG. 11. Photograph of the internal components of the premagnetization pulse generator constructed for resetting the ferromagnetic cores on BLUE. This photograph shows the generator's four positive and four negative output channels. The dark blue 3D-printed plastic structures on top of the capacitors are the screw-in breakers for connecting individual channels. In this photograph, only the cables to the first cavity are attached.

operation of a bipolar LTD brick. In each channel, the resulting output pulse of the $1-\mathrm{mF}$ capacitor is sent through a short length of nichrome wire acting as a high-power $250-\mathrm{m} \Omega$ resistor, over-damping the pulse, before it is sent to the cavity. An internal photograph of the premagnetization pulse generator constructed for BLUE is shown in Fig. 11.

Output current pulses from a single channel of the premagnetization pulse generator were measured with a Pearson coil (see Fig. 12). When the cores have already been saturated in the desired direction (i.e., the pre-mag generator has already been successfully fired in preparation for an LTD discharge), the pre-mag current pulse into the cavity is nearly identical to the pulse into a short-circuit load, indicating that the cavity has a very low impedance for the direction of the pre-mag current pulse (and thus a high impedance for the direction of the parasitic current pulse during an LTD discharge). When the cores are saturated in the undesired direction (i.e., the cores are in need of a resetting pre-mag pulse), a notch in the waveform of the pre-mag current is observed (see Fig. 12). This notch is the result of the initially high impedance of the cavity (for the direction of the pre-mag current) dropping rapidly in the first $\sim 25 \mu$ s of the pulse. Note that $25 \mu$ s is consistent with the rough estimate of $33 \mu$ s from the volt-second product of the cores. This notch was predicted via LTspice modeling prior to the first experimental tests of the system. The LTspice pre-mag model was then fine-tuned to nearly replicate the experimental results, as shown in Fig. 12. The dynamic impedance seen by the pre-mag generator is

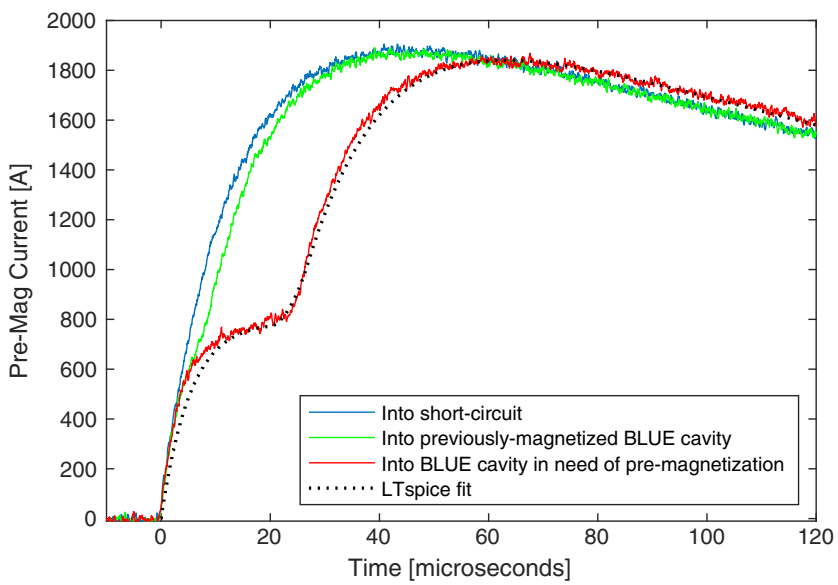

FIG. 12. Output current waveforms from a single channel of the pre-magnetization pulse generator into three load scenarios: (1) into a short-circuit load (blue); (2) into the cavity for the case where the cores have already been reset or pre-magnetized (green); and (3) into the cavity for the case where the cores need to be reset or pre-magnetized (red). Also shown is a waveform produced by an LTspice model fine-tuned to closely match the experimental results.

both inductive and resistive, with the resistive component being due to the eddy current effect mentioned previously. However, only the resistive component was tuned (modulated in time) to nearly replicate the experimental results with LTspice. Note that a tuned inductance (modulated in time) could also have been used to obtain results that agree well with experiments, so this tuning method does not necessarily provide the exact temporal behavior of the cores' effective inductance or resistance values.

The effects of successful pre-magnetization on the main LTD cavity discharge can be seen in load current measurements taken when the cavity is fired into a resistive load. This is discussed in Sec. IV B. In general, premagnetization of the cavity increases the amplitude of the LTD's main discharge current by $10-20 \%$ and extends the duration of positive current into the load.

There is a large issue to be aware of in designing a premagnetization pulse generator for LTDs. Since (ideally) the pre-mag cables remain attached to the cavity during an LTD discharge, the high-voltage, high-frequency pulse from the main discharge can travel back to the pre-mag generator, potentially damaging medium-voltage electronics such as the charging diodes or, more importantly, the thyristor. Two thyristors on BLUE's pre-mag system were damaged during LTD discharges, despite attempts to protect them with metaloxide varistors and an over-voltage spark-gap placed directly across the thyristor terminals (see Fig. 10). Transientvoltage-suppression diodes and/or better spark-gaps may provide more effective protection from the fast transients, but this has yet to be demonstrated experimentally. For now, triggered spark-gaps are recommended as a more robust alternative to thyristors for a pre-mag system switch. 


\section{RESISTIVE LOAD TESTING OF THE PROTOTYPE BLUE CAVITY}

\section{A. Bipolar Marx generator configuration}

The prototype cavity was first tested with a polycarbonate lid, as shown in Figs. 1(a) and 2. This allows for internal visual access during testing. It also eliminates the parasitic current path and thus the need for ferromagnetic cores. This effectively puts the cavity into a bipolar Marx generator architecture, rather than a LTD architecture. This coreless configuration is closely related to an LTD spinoff concept known as the impedance-matched Marx generator (IMG) [27].

A dummy resistive load for the cavity was fabricated using an arrangement of spare ceramic resistors. The resistance of the load was approximately $1 \Omega$, while the inductance was estimated to be greater than $100 \mathrm{nH}$. An image of the cavity mounted on its side (i.e., mounted in the white, cavity-stacking support rack) and firing into the resistive load is shown in Fig. 13.

A current viewing resistor (CVR) was installed in series with the resistive load to obtain calibrated load current data. However, the anode (front or top) side of BLUE's load region must be grounded for an oscilloscope to properly monitor the CVR signal. This became problematic with the polycarbonate lid installed on the cavity's front or top side, because in this case, the cathode becomes the grounded end of BLUE's load region. This occurs because BLUE's central cathode stalk is in direct contact with the metal lid

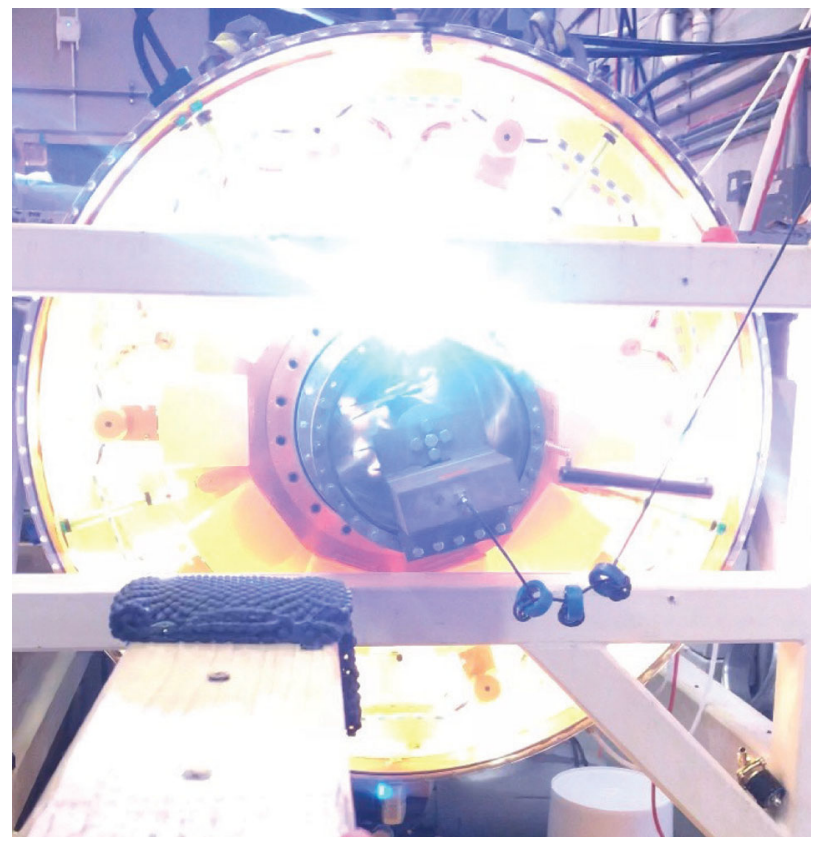

FIG. 13. The prototype BLUE cavity mounted on its side and firing into a resistive load in the bipolar Marx generator configuration (i.e., using the clear plastic lid and thus not using ferromagnetic cores). The bright flash is an undesirable arc to the white, steel support frame. on the back (bottom) side of the cavity, which is in contact with the bulk of the metal cavity, the vacuum pumps, and the grounded steel frame that supports the cavity. Operating the cavity in this configuration also resulted in troublesome arcing from the anode to the steel frame (see Fig. 13). As a result of these issues, the data acquired from the CVR was garbled and not representative of the true load current. For this reason, current measurements are not presented for the cavity in the bipolar Marx configuration.

The aforementioned issues could be remedied by placing the polycarbonate lid on the back side of the cavity and isolating the cathode from the vacuum pump systems with a ceramic break. Other coreless configurations that could work include those described in Refs. [27,28]. However, to avoid delays in commissioning the BLUE facility, the decision was made to move on to testing the cavity in its original LTD configuration-i.e., replacing the polycarbonate lid on the cavity's front side with the original steel lid, installing the ferromagnetic cores, and implementing the core reset or pre-mag system.

\section{B. LTD configuration}

Replacing the polycarbonate lid with the original steel lid and inserting the ferromagnetic cores transforms the cavity back into the traditional LTD configuration [see Fig. 1(b)]. The pre-mag pulse generator is now desired to magnetize the cores in the proper direction prior to an LTD shot, thus minimizing the current taking the parasitic path (see Sec. III E).

A new, higher quality resistive load (using new HVR APC ceramic disk resistors) was fabricated for BLUE in the LTD architecture. One of the motivations for doing this was that the simple cylindrical geometry of these disk resistors allows the load inductance to be more precisely estimated. The new load was also designed to have a variable

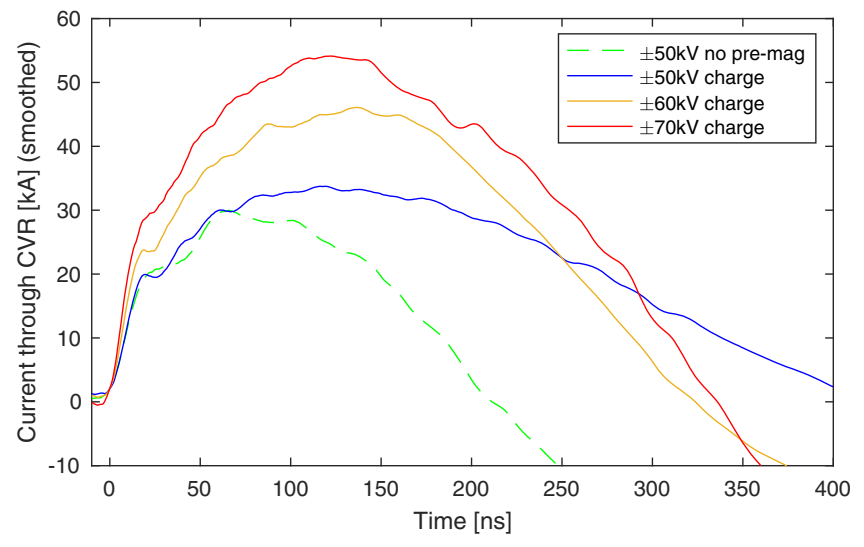

FIG. 14. Nominal experimental current traces from the prototype BLUE cavity configured as an LTD at different charge voltages, acquired using a current-viewing resistor (CVR). The data have been smoothed to reduce high-frequency noise. The resistive load has resistance and inductance values of approximately $1.5 \Omega$ and $110 \mathrm{nH}$, respectively. 


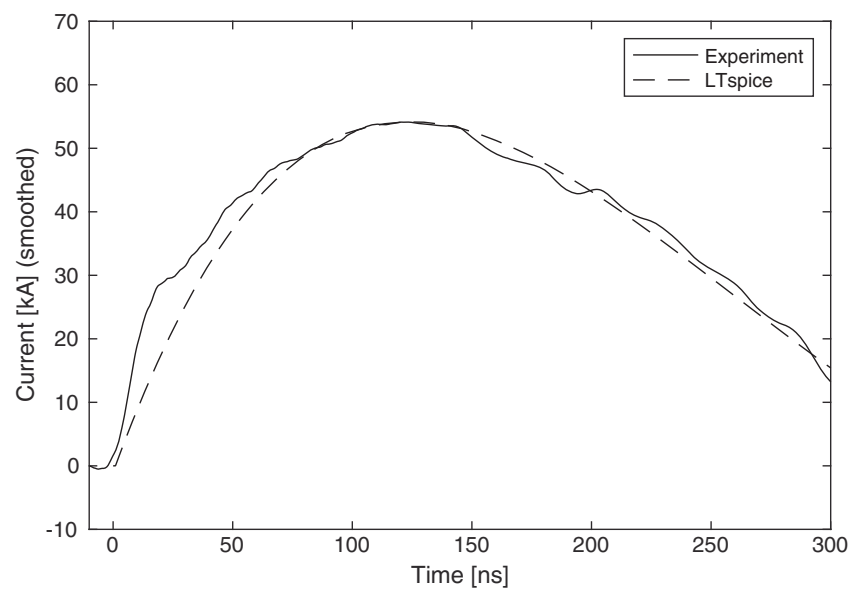

FIG. 15. Experimental current trace from the prototype BLUE cavity configured as an LTD at $\pm 70 \mathrm{kV}$ charge voltage into a resistive and inductive load. The experimental curve has been smoothed to remove high-frequency noise. Also plotted for comparison is the current trace from an LTspice simulation of the discharge. In the simulation, the load resistance was $1.5 \Omega$ and the load inductance was $110 \mathrm{nH}$, which is in good agreement with the estimated load resistance and inductance based on geometry.

resistance of $0.5-4 \Omega$ by stacking multiple disk resistors in series. For the experiments reported herein, the load resistance was $1.5 \Omega$, and the load inductance was estimated to be $\sim 110 \mathrm{nH}$.

Figures 14 and 15 show experimental current traces from the prototype BLUE cavity in the LTD configuration. These traces were acquired by the CVR. Figure 14 shows the current traces at varying charge voltages: $\pm 50 \mathrm{kV}$, $\pm 60 \mathrm{kV}$, and $\pm 70 \mathrm{kV}$. Figure 14 also shows the effects of pre-magnetization on the load current. Without premagnetization, the load current is smaller in amplitude and shorter in duration, as significant current begins to leak into the parasitic current path around the time of peak current.

Figure 15 shows good agreement between an experimentally measured current trace and the current trace generated by an LTspice simulation of the discharge. In this case, the charge voltage was $\pm 70 \mathrm{kV}$. The larger

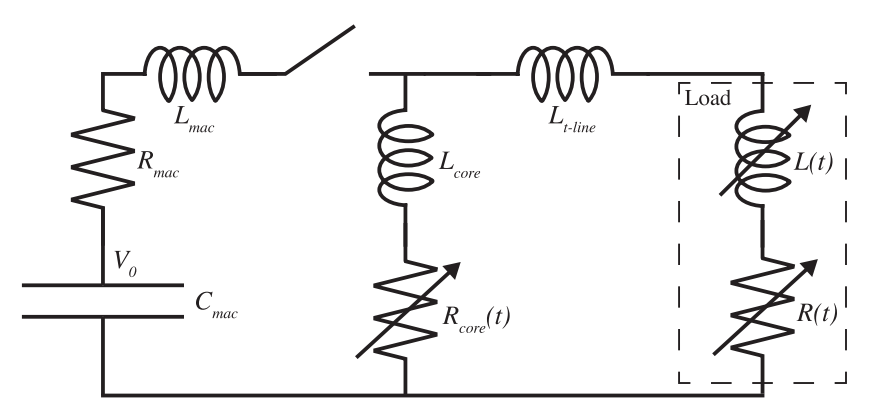

FIG. 16. A simple circuit model of the BLUE LTD facility. Formulas for the circuit elements, along with approximate values for a single cavity, are provided in Table I.
TABLE I. Formulas for the simple circuit elements shown in Fig. 16. The approximate values provided in the rightmost column are for a single cavity-i.e., with $N_{\text {cav }}=1$. $L_{\text {t-line }}$ is the inductance of the transmission line to the load, which can vary depending on the installed hardware. A transmission line to the chamber procured for $Z$-pinch experiments has an inductance of $\approx 16 \mathrm{nH}$. $Z_{\mathrm{mac}}$ is the machine impedance.

\begin{tabular}{lcc}
\hline \hline $\begin{array}{l}\text { Circuit } \\
\text { variable }\end{array}$ & Formula & $\begin{array}{c}\text { Approximate value } \\
\text { for } N_{\text {cav }}=1\end{array}$ \\
\hline$C_{\text {mac }}$ & $5 C_{\text {cap }} / N_{\text {cav }}$ & $100 \mathrm{nF}$ \\
$V_{0}$ & $2 \mid V_{\text {charge }} \times N_{\text {cav }}$ & $200 \mathrm{kV} \mathrm{max}$ \\
$R_{\text {mac }}$ & $R_{\text {brick }} N_{\text {cav }} / 10$ & $4-60 \mathrm{~m} \Omega$ \\
$L_{\text {mac }}$ & $L_{\text {brick }} N_{\text {cav }} / 10$ & $22 \mathrm{nH}$ \\
$L_{\text {core }}$ & $L_{0, \text { core }} N_{\text {cav }}$ & $950 \mathrm{nH}$ \\
$R_{\text {core }}(t)$ & $R_{0, \text { core }}(t) \times N_{\text {cav }}$ & $0.50 \Omega$ for $t \ll 10 \mu \mathrm{s})$ \\
$L_{\text {t-line }}$ & $L_{\mathrm{t}-\text { line }}$ & $\sim 16 \mathrm{nH}$ for chamber loads \\
$Z_{\text {mac }}$ & $\sqrt{L_{\text {mac }} / C_{\text {mac }}}$ & $0.47 \Omega$ \\
\hline \hline
\end{tabular}

amplitude of the experimental trace before $t \approx 80 \mathrm{~ns}$ is likely due to imperfect modeling of the core behavior and thus current losses to the parasitic current path.

From these initial calibration and pre-magnetization experiments, a simple circuit model was semiempirically developed for BLUE. The model is illustrated schematically in Fig. 16. The values to be used in the circuit model are provided in Table I. In the central column of Table I, formulas are provided for calculating the circuit element values as a function of the number of cavities, $N_{\text {cav }}$. In the rightmost column of Table I, approximate values are given for a single cavity-i.e., with $N_{\text {cav }}=1$.

\section{TESTING A MILO ON BLUE}

The first physics load to be mounted on the prototype BLUE cavity was a HPM device known as a MILO [29]. An LTD paired with such a device can represent a very compact HPM source, desirable in many applications. To our knowledge, Ref. [30] presents the first demonstration of a compact LTD being used to drive an HPM source-in this case, the HPM source was a coaxial vircator.

The MILO driven with BLUE is a crossed-field HPM device [31] that, in this case, operates in a coaxial, axisymmetric geometry [see Fig. 17(a)]. In a MILO, the drive current produces a magnetic field that insulates the anode from electrons emitted at the cathode. Thus, external magnetic field coils are not required to operate a MILO, as they are in the case of other crossed-field devices (e.g., magnetrons). For the cylindrical MILO shown in Fig. 17, the insulating magnetic field is azimuthal, $\mathbf{B}=B_{\theta} \hat{\boldsymbol{\theta}}$, and the driving electric field is radial $\mathbf{E}=E_{r} \hat{\boldsymbol{r}}$. Electrons emitted radially outward from the velvet-covered central cathode are turned by the magnetic field and begin to drift axially through the periodic slow wave structure (SWS) at a velocity of $\mathbf{v}_{\text {drift }}=\mathbf{E} \times \mathbf{B} / B^{2}$ [see Fig. 17(b)]. 

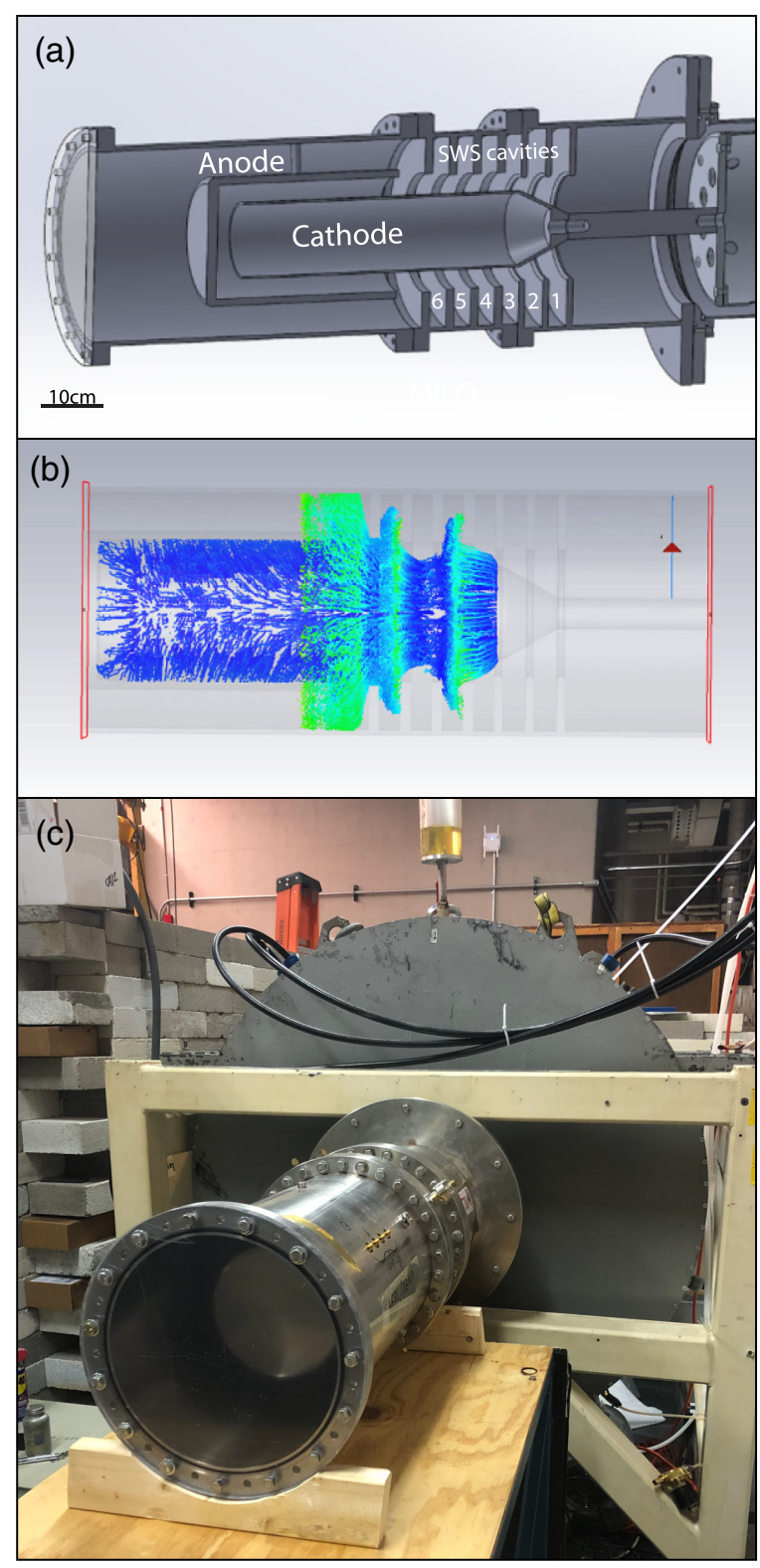

FIG. 17. (a) Cross-section of the MILO HPM device. (b) CST particle-in-cell simulation of the MILO in $\pi$-mode operation, with the simulated electrons (blue or green) bunching into well-formed spoke-like structures in the SWS [32]. (c) The MILO mounted to the prototype BLUE cavity.

The MILO mounted to BLUE is shown in Fig. 17(c). This MILO was previously tested by the Air Force Research Laboratory (AFRL) in Albuquerque, NM [33-36]. In these tests, the MILO was driven by the Sandia National Laboratories $500-\mathrm{kV}, 5-\Omega, 300-600-\mathrm{ns}$ IMP pulser $[35,36]$. Note that the IMP pulser has remarkably similar drive characteristics to the full 4-cavity BLUE facility. Operation of this MILO has been simulated recently by D. A. Packard et al. [32] [see also Fig. 17(b)].

While the specific details of our BLUE MILO experiments are left for a future publication, the preliminary
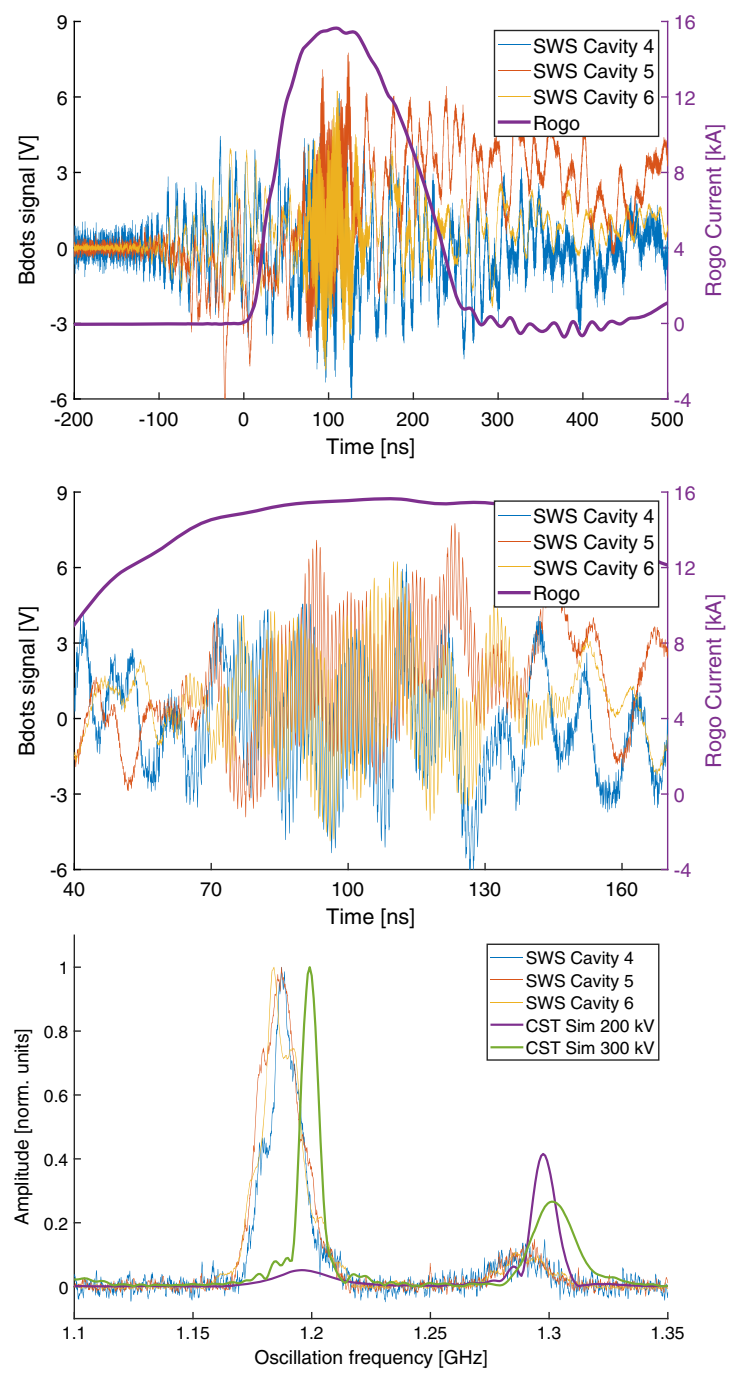

FIG. 18. Voltage signals from three $B$-dot probes in the slowwave structure of the MILO during a discharge of the BLUE prototype cavity with a charge voltage of $\pm 70 \mathrm{kV}$ (top and middle). Also plotted is the load current into the MILO, which was measured using a calibrated Rogowski coil. Microwave oscillations at a frequency of $1.187 \mathrm{GHz}$ are observed for approximately $80 \mathrm{~ns}$ (during the period of 65-145 ns). The oscillations are well-sampled for Fourier analysis and comparison with simulation (bottom). Plotted are smoothed, normalized FFTs of the $B$-dot data, as well as normalized FFTs from CST simulations in Ref. [32] at different drive voltages.

demonstration of microwave generation is provided here. In Fig. 18, voltage signals are presented from three $B$-dot probes located in the SWS of the MILO. Also shown is a Rogowski coil signal, integrated and calibrated to give the total current entering the MILO. The charge voltage for the shot was $\pm 70 \mathrm{kV}$ (140 kV open circuit). Microwave oscillations at a frequency of $1.187-\mathrm{GHz}$ are observed for approximately $80 \mathrm{~ns}$ (during the period of 65-145 ns).

The oscillations are well sampled for Fourier analysis and are distinct from background noise. Plotted in the bottom pane of Fig. 18 are smoothed, normalized fast 
Fourier transforms (FFTs) of the $B$-dot data, as well as normalized FFTs from CST Studio Suite simulations at different drive voltages [32]. The experimental frequency of $1.187 \mathrm{GHz}$ is consistent with beam-loaded $\pi$-mode operation in these particle-in-cell simulations. The differences between the experimental and simulated FFTs are due primarily to imperfect experimental geometries (leading to slight differences in the center frequencies of the FFT peaks) and disparate sampling windows (leading to differences in the widths of the FFT peaks). Regarding the disparate sampling windows, the simulations produced oscillations for $\sim 150 \mathrm{~ns}$, whereas the experiments produced oscillations for $80 \mathrm{~ns}$. This results in the simulated FFTs having narrower peaks than the experimental FFTs, due to the uncertainties associated with the time-bandwidth product [37].

The excitation of the oscillations in the experiment for approximately $80 \mathrm{~ns}$ is remarkable given the relatively lowdrive voltage applied $\left(V_{0}=2\left|V_{\text {charge }}\right|=140 \mathrm{kV}\right)$ and the very low driver impedance of just a single BLUE LTD cavity $(0.5 \Omega)$. In simulation, similar oscillations were predicted only at higher voltages (see Fig. 18). Future experiments are planned to characterize the rf output power as a function of the applied drive voltage. The drive voltage will be varied (increased) by increasing the number of BLUE cavities stacked together in series, since $V_{0}=$ $2\left|V_{\text {charge }}\right| \times N_{\text {cav }}$ (see Table I).

\section{SUMMARY}

The first prototype cavity of the BLUE LTD facility at the University of Michigan is now operational. The components to make three more identical cavities have been procured, and construction of the second cavity has begun. A modular system like BLUE will allow future studies of LTD cavity coupling and pulse shaping $[8,14]$. This informs the design of next generation LTD-based super-accelerators like Z-300 and Z-800 [15], which could likely demonstrate ignition of a nuclear fusion target using the MagLIF concept [38].

Beyond studies of pulsed-power technology, BLUE is capable of driving a wide variety of physics loads. A MILO HPM source has been successfully driven to produce microwaves, and a large diameter vacuum chamber with many diagnostic ports has been prepared to house various $Z$-pinch and $X$-pinch loads. The authors are excited to offer this new facility and capability to the pulsed-power, HPM, and HEDP communities.

\section{ACKNOWLEDGMENTS}

The authors would like to thank Mike Cuneo, Steve Exelby, Cayetano Wagner, and Mark Perrault for their assistance with transferring the four Ursa-Minor/BLUE LTD cavities from SNL to UM. The authors would further like to thank Mark Perrault for his ongoing technical and electrical assistance in the construction of BLUE sub-systems. The authors would also like to thank all of the PPML students for providing invaluable insights in working with LTD technology and high voltage, and for providing assistance in the construction of BLUE. Additionally, the authors thank Simon Bland for his consultation in development of the PT003 replacement pulse module. This work was supported in part by the U.S. Office of Naval Research through the Young Investigator Program under Grant No. N00014-18-1-2499, in part by the U.S. Air Force Office of Scientific Research through the MURI program under Grant No. FA9550-20-1-0409, in part by the NNSA Stewardship Sciences Academic Programs under DOE Cooperative Agreement DE-NA0003764, and in part by Sandia National Laboratories through the StevensonWydler Gift Program. Sandia is a multimission laboratory managed and operated by National Technology and Engineering Solutions of Sandia, LLC., a wholly owned subsidiary of Honeywell International, Inc., for the NNSADOE under Contract No. DE-NA0003525. Approved for public release; distribution is unlimited. Public Affairs release approval \#AFRL-2021-2304. This paper describes objective technical results and analysis. Any subjective views or opinions that might be expressed in the paper do not necessarily represent the views of the U.S. Department of Energy or the United States Government.

[1] B. M. Koval'chuk, V. A. Vizir', A. A. Kim, E. V. Kumpyak, S. V. Loginov, A. N. Bastrikov, V. V. Chervyakov, N. V. Tsoi, P. Monjaux, and D. Kh'yui, Fast primary storage device utilizing a linear pulse transformer, Russ. Phys. J. 40, 1142 (1997).

[2] A. A. Kim, B. M. Kovalchuk, E. V. Kumpjak, and N. V. Zoi, 0.75 MA, $400 \mathrm{~ns}$ rise time LTD stage, in Proceedings of 12th IEEE International Pulsed Power Conference (1999) (Cat. No. 99CH36358), Vol. 2, pp. 955-958.

[3] M. G. Mazarakis and R. B. Spielman, A compact, highvoltage e-beam pulser, in Proceedings of 12th IEEE International Pulsed Power Conference (1999) (Cat. No. 99CH36358), Vol. 1, pp. 412-415.

[4] A. A. Kim and B. M. Kovalchuk, High power direct driver for Z-pinch loads, in Proceedings of the 12th Symposium on High Current Electronics (part of the First International Congress on Radiation Physics, High Current Electronics, and Modification of Materials), edited by G. Mesyats, B. Kovalchuk, and G. Remnev (Institute of High Current Electronics, Tomsk, Russia, 2000), p. 263.

[5] W. A. Stygar, M. E. Cuneo, D. I. Headley, H. C. Ives, R. J. Leeper, M. G. Mazarakis, C. L. Olson, J. L. Porter, T. C. Wagoner, and J. R. Woodworth, Architecture of petawattclass z-pinch accelerators, Phys. Rev. ST Accel. Beams 10, 030401 (2007).

[6] A. A. Kim, M. G. Mazarakis, V. A. Sinebryukhov, B. M. Kovalchuk, V. A. Visir, S. N. Volkov, F. Bayol, A. N. Bastrikov, V. G. Durakov, S. V. Frolov, V. M. Alexeenko, D. H. McDaniel, W. E. Fowler, K. LeChien, C. Olson, W. A. Stygar, K. W. Struve, J. Porter, and R. M. Gilgenbach, 
Development and tests of fast 1-MA linear transformer driver stages, Phys. Rev. ST Accel. Beams 12, 050402 (2009).

[7] R. M. Gilgenbach, M. R. Gomez, J. C. Zier, W. W. Tang, D. M. French, Y. Y. Lau, M. G. Mazarakis, M. E. Cuneo, M. D. Johnston, B. V. Oliver, T. A. Mehlhorn, A. A. Kim, and V. A. Sinebryukhov, MAIZE: A 1 MA LTD-Driven Z-Pinch at The University of Michigan, AIP Conf. Proc. 1088, 259 (2009).

[8] W. A. Stygar, W. E. Fowler, K. R. LeChien, F. W. Long, M. G. Mazarakis, G. R. McKee, J. L. McKenney, J. L. Porter, M. E. Savage, B. S. Stoltzfus, D. M. Van De Valde, and J. R. Woodworth, Shaping the output pulse of a lineartransformer-driver module, Phys. Rev. ST Accel. Beams 12, 030402 (2009).

[9] M. G. Mazarakis, W. E. Fowler, A. A. Kim, V. A. Sinebryukhov, S. T. Rogowski, R. A. Sharpe, D. H. McDaniel, C. L. Olson, J. L. Porter, K. W. Struve, W. A. Stygar, and J. R. Woodworth, High current, 0.5-MA, fast, 100-ns, linear transformer driver experiments, Phys. Rev. ST Accel. Beams 12, 050401 (2009).

[10] M. G. Mazarakis et al., High-current linear transformer driver development at Sandia National Laboratories, IEEE Trans. Plasma Sci. 38, 704 (2010).

[11] A. A. Kim, M. G. Mazarakis, V. I. Manylov, V. A. Vizir, and W. A. Stygar, Energy loss due to eddy current in linear transformer driver cores, Phys. Rev. ST Accel. Beams 13, 070401 (2010).

[12] J. Leckbee, S. Cordova, B. Oliver, T. Webb, M. Toury, M. Caron, R. Rosol, B. Bui, T. Romero, and D. Ziska, Linear transformer driver (LTD) research for radiographic applications, in Proceedings of 2011 IEEE Pulsed Power Conference (IEEE, Chicago, 2011), pp. 614-618.

[13] J. J. Leckbee, T. D. Pointon, S. R. Cordova, B. V. Oliver, T. J. Webb, M. Toury, M. Caron, and D. W. Droemer, Commissioning and power flow studies of the 2.5-MeV Ursa Minor LTD, in Proceedings of 2012 IEEE International Power Modulator and High Voltage Conference (IPMHVC) (IEEE, San Diego, 2012), pp. 169-173.

[14] A. A. Kim, M. G. Mazarakis, V. A. Sinebryukhov, S. N. Volkov, S. S. Kondratiev, V. M. Alexeenko, F. Bayol, G. Demol, and W. A. Stygar, Square pulse linear transformer driver, Phys. Rev. ST Accel. Beams 15, 040401 (2012).

[15] W. A. Stygar et al., Conceptual designs of two petawattclass pulsed-power accelerators for high-energy-densityphysics experiments, Phys. Rev. ST Accel. Beams 18, 110401 (2015).

[16] L. Zhou, Z. Li, Z. Wang, C. Liang, M. Li, J. Qi, and Y. Chu, Design of a 5-MA 100-ns linear-transformer-driver accelerator for wire array Z-pinch experiments, Phys. Rev. Accel. Beams 19, 030401 (2016).

[17] A. Kim, B. Kovalchuk, V. Kokshenev, A. Shishlov, N. Ratakhin, V. Oreshkin, V. Rostov, V. Koshelev, and V. Losev, Review of high-power pulsed systems at the Institute of High Current Electronics, Matter Radiat. Extremes 1, 201 (2016).

[18] P. C. Campbell, J. M. Woolstrum, F. Antoulinakis, T. M. Jones, D. A. Yager-Elorriaga, S. M. Miller, N. M. Jordan, Y. Y. Lau, R. M. Gilgenbach, and R. D. McBride, Diagnostic and power feed upgrades to the MAIZE facility, IEEE Trans. Plasma Sci. 46, 3973 (2018).
[19] R. D. McBride et al., A primer on pulsed power and linear transformer drivers for high energy density physics applications, IEEE Trans. Plasma Sci. 46, 3928 (2018).

[20] J. D. Douglass et al., $100 \mathrm{GW}$ linear transformer driver cavity: Design, simulations, and performance, Phys. Rev. Accel. Beams 21, 120401 (2018).

[21] A. A. Kim and M. G. Mazarakis, The Story of the LTD Development, IEEE Trans. Plasma Sci. 48, 749 (2020).

[22] F. Conti, J. C. Valenzuela, V. Fadeev, N. Aybar, D. B. Reisman, A. Williams, G. Collins, J. Narkis, M. P. Ross, F. N. Beg, and R. B. Spielman, MA-class linear transformer driver for Z-pinch research, Phys. Rev. Accel. Beams 23, 090401 (2020).

[23] J. Woodworth, J. Alexander, F. Gruner, W. Stygar, M. Harden, J. Blickem, G. Dension, F. White, L. Lucero, H. Anderson et al., Low-inductance gas switches for linear transformer drivers, Phys. Rev. ST Accel. Beams 12, 060401 (2009).

[24] J. R. Woodworth, W. A. Stygar, L. F. Bennett, M. G. Mazarakis, H. Anderson, M. Harden, J. Blickem, F. Gruner, and R. White, New low inductance gas switches for linear transformer drivers, Phys. Rev. ST Accel. Beams 13, 080401 (2010).

[25] J. Yan, S. Parker, T. Gheorghiu, N. Schwartz, S. Theocharous, and S. N. Bland, Miniature solid-state switched spiral generator for the cost effective, programmable triggering of large scale pulsed power accelerators, Phys. Rev. Accel. Beams 24, 030401 (2021).

[26] A. A. Kim, A. N. Bastrikov, S. N. Volkov, V. G. Durakov, B. M. Kovalchuk, and V. A. Sinebryukhov, Development of the ultra-fast LTD stage, in Proceedings of 2002 14th International Conference on High-Power Particle Beams (BEAMS) (IEEE, Albuquerque, 2002), Vol. 1, pp. 81-84.

[27] W. Stygar, K. LeChien, M. Mazarakis, M. Savage, B. Stoltzfus, K. Austin, E. Breden, M. Cuneo, B. Hutsel, S. Lewis et al., Impedance-matched Marx generators, Phys. Rev. Accel. Beams 20, 040402 (2017).

[28] P.-A. Gourdain, M. Evans, P. Efthimion, R. Ellis, W. Fox, H. R. Hasson, H. Ji, R. V. Shapovalov, J. R. Young, and I. West-Abdallah, Coreless fast pulsed-power drivers, IEEE Trans. Plasma Sci. 49, 2161 (2021).

[29] R. W. Lemke and M. C. Clark, Theory and simulation of high-power microwave generation in a magnetically insulated transmission line oscillator, J. Appl. Phys. 62, 3436 (1987).

[30] B. M. Kovalchuk, S. D. Polevin, R. V. Tsygankov, and A. A. Zherlitsyn, S-band coaxial vircator with electron beam premodulation based on compact linear transformer driver, IEEE Trans. Plasma Sci. 38, 2819 (2010).

[31] J. Benford, J. A. Swegle, and E. Schamiloglu, High Power Microwaves, 2nd ed. (Taylor \& Francis Group, London, 2007).

[32] D. A. Packard, A. Cooleybeck, N. M. Jordan, B. J. Sporer, A. E. Mazarakis, Y. Lau, R. M. Gilgenbach, and R. D. McBride, HFSS and CST Simulations of a GW-Class MILO, IEEE Trans. Plasma Sci. 48, 1894 (2020).

[33] S. E. Calico, M. C. Clark, R. W. Lemke, and M. C. Scott, Experimental and theoretical investigations of a magnetically insulated line oscillator (MILO), in Proceedings of Intense Microwave Pulses III, edited by $\mathrm{H}$. E. Brandt, 
International Society for Optics and Photonics (SPIE, San Diego, 1995), Vol. 2557, pp. 50-59.

[34] R. W. Lemke, S. E. Calico, and M. C. Clark, Investigation of a load-limited, magnetically insulated transmission line oscillator (MILO), IEEE Trans. Plasma Sci. 25, 364 (1997).

[35] M. Haworth, G. Baca, J. Benford, T. Englert, K. Hackett, K. Hendricks, D. Henley, M. LaCour, R. Lemke, D. Price et al., Significant pulse-lengthening in a multigigawatt magnetically insulated transmission line oscillator, IEEE Trans. Plasma Sci. 26, 312 (1998).
[36] M. Haworth, J. Luginsland, and R. Lemke, Evidence of a new pulse-shortening mechanism in a load-limited MILO, IEEE Trans. Plasma Sci. 28, 511 (2000).

[37] D. Gabor, Theory of communication. part 1: The analysis of information, J. Inst. Electr. Eng. 93, 429 (1946).

[38] S. Slutz, W. Stygar, M. Gomez, K. Peterson, A. Sefkow, D. Sinars, R. Vesey, E. Campbell, and R. Betti, Scaling magnetized liner inertial fusion on $\mathrm{Z}$ and future pulsed-power accelerators, Phys. Plasmas 23, 022702 (2016). 